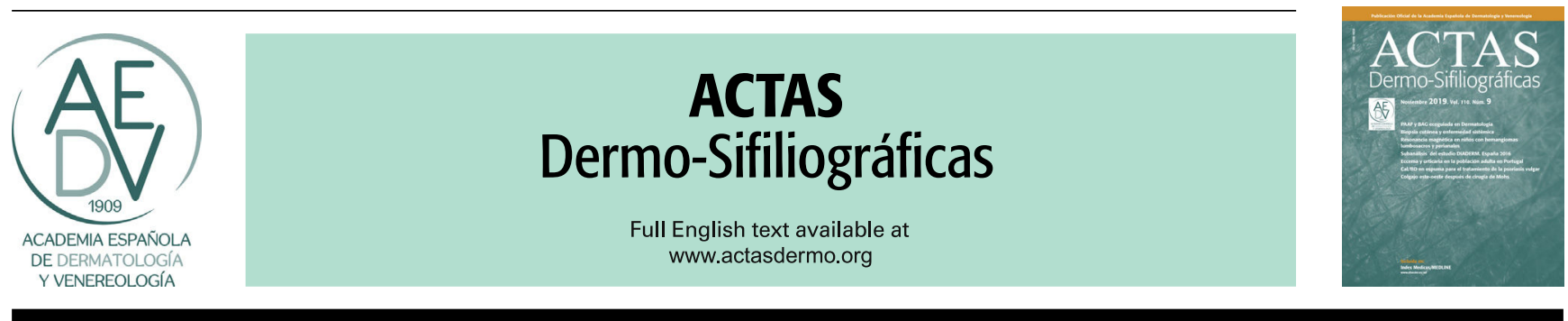

\title{
Guía para el diagnóstico, el tratamiento y la prevención del eccema de manos
}

\section{J.F. Silvestre Salvador ${ }^{\mathrm{a}, *}, \mathrm{~F}$. Heras Mendaza ${ }^{\mathrm{b}}$, M. Hervella Garcés ${ }^{\mathrm{c}}$, D. Palacios-Martínez ${ }^{\mathrm{d}}$, R. Sánchez Camacho ${ }^{\mathrm{e}}$, R. Senan Sanz ${ }^{\mathrm{f}}$, A. Apellaniz González ${ }^{\mathrm{g}}$ y A.M. Giménez-Arnau ${ }^{\mathrm{h}}$}

\author{
a Servicio de Dermatología, Hospital General Universitario de Alicante, Alicante, España \\ b Servicio de Dermatología, Hospital Universitario Fundación Jiménez Díaz, Madrid, España \\ c Servicio de Dermatología, Complejo Hospitalario de Navarra, Pamplona, España \\ ' Medicina de Atención Primaria, Centro de Salud Isabel II, Parla, Madrid, España \\ e Medicina General, Centro de Salud Ávila Rural, Ávila, España \\ ${ }^{\mathrm{f}}$ Equipo de Atención Primaria el Clot, Instituto Catalán de la Salud, Barcelona, España \\ ` Departamento de Estomatología, Facultad de Medicina y Enfermería, Euskal Herriko Unibertsitatea-Universidad del País Vasco, \\ Leioa, Bizkaia, España \\ ${ }^{\text {h }}$ Departamento de Dermatología, Hospital del Mar, Universitat Autónoma de Barcelona, Barcelona, España
}

Recibido el 7 de marzo de 2019; aceptado el 21 de abril de 2019

\section{PALABRAS CLAVE \\ Eccema crónico; \\ Dermatitis; \\ Emolientes; \\ Corticoides tópicos; \\ Médico de atención \\ primaria; \\ Manos; \\ Diagnóstico; \\ Criterios de \\ derivación}

\section{KEYWORDS}

Chronic eczema;

Dermatitis;

Emollients;

Topical

\begin{abstract}
Resumen El eccema de manos es una patología frecuente con un fuerte impacto en la calidad de vida de los pacientes y un alto coste social y laboral. Su manejo por los médicos de atención primaria y de medicina del trabajo es complejo debido a la variedad de etiologías, la evolución difícilmente predecible de la enfermedad y la respuesta al tratamiento. El diagnóstico precoz y las medidas protectoras adecuadas son esenciales para evitar la cronificación, que es mucho más difícil de tratar. Una correcta derivación a un especialista y la valoración de una baja laboral en el momento adecuado resultan cruciales para un buen manejo de estos pacientes.

En esta guía sobre el eccema crónico de manos analizamos el proceso diagnóstico, las medidas preventivas y los tratamientos, con especial énfasis en el papel del médico de atención primaria y de medicina del trabajo en los estados iniciales de su manejo.

(C) 2019 AEDV. Publicado por Elsevier España, S.L.U. Este es un artículo Open Access bajo la licencia CC BY-NC-ND (http: //creativecommons.org/licenses/by-nc-nd/4.0/).
\end{abstract}

\section{Guidelines for the Diagnosis, Treatment, and Prevention of Hand Eczema}

Abstract Hand eczema is a common condition associated with significantly impaired quality of life and high social and occupational costs. Managing hand eczema is particularly challenging for primary care and occupational health physicians as the condition has varying causes and

\footnotetext{
* Autor para correspondencia.

Correo electrónico: silvestre_jfr@gva.es (J.F. Silvestre Salvador).
} 
corticosteroids;

General

practitioners;

Hands;

Diagnosis;

Referral criteria both disease progression and response to treatment are difficult to predict. Early diagnosis and appropriate protective measures are essential to prevent progression to chronic eczema, which is much more difficult to treat. Appropriate referral to a specialist and opportune evaluation of the need for sick leave are crucial to the good management of these patients.

These guidelines cover the diagnosis, prevention, and treatment of chronic hand eczema and highlight the role that primary care and occupational health physicians can play in the early management of this disease.

(C) 2019 AEDV. Published by Elsevier España, S.L.U. This is an open access article under the CC BY-NC-ND license (http://creativecommons.org/licenses/by-nc-nd/4.0/).

\section{Introducción}

El eccema de manos (EM) es una patología con una alta prevalencia, estimada en torno al $10 \%$ de la población general ${ }^{1}$. El $80 \%$ de las dermatosis profesionales son EM, y constituyen una causa frecuente de baja en el entorno laboral ${ }^{2}$. No obstante, en España no existe una guía nacional para el diagnóstico, la prevención y el tratamiento de esta patología. En 2015 se publicaron las Guías europeas para el diagnóstico, prevención, protección y tratamiento del eccema crónico de manos $(E C M)^{3}$, pero dichas guías no cubren la complejidad existente entre los distintos niveles del sistema sanitario español. En España el primer contacto del paciente con EM dentro del sistema sanitario público suele ser el médico de atención primaria (AP), pero al no haber una guía nacional, los criterios de actuación y derivación al especialista no están estandarizados. La complejidad de este esquema se ve agravada por la diversidad de los modelos sanitarios regionales.

El manejo del EM requiere una actuación precoz de diagnóstico y tratamiento para evitar su posible cronificación, algo que requiere la coordinación entre los distintos niveles asistenciales. Por ello se justifica la necesidad de tener sistematizados los criterios respecto a cómo el paciente con EM debe ser tratado en AP, cuándo debe ser derivado al especialista, cómo debe ser el seguimiento del paciente y cuándo es necesaria la interlocución con las mutuas laborales y los médicos del trabajo. La Academia Española de Dermatología y Venereología (AEDV), a través del Grupo Español de Investigación en Dermatitis de Contacto y Alergia Cutánea (GEIDAC), decidió desarrollar este documento de revisión actualizado que sirviera como base para la evaluación diagnóstica, el tratamiento y el seguimiento, así como para establecer criterios unificados y consensuados de derivación de pacientes con EM cuando la evolución de la enfermedad requiere ser valorada en un segundo nivel asistencial.

Con el liderazgo de la Academia, la preparación de este documento ha contado con la participación de la Sociedad Española de Médicos de Atención Primaria (SEMERGEN), la Sociedad Española de Médicos de Familia y Comunitaria (semFYC), la Sociedad Española de Médicos Generales y de Familia (SEMG) y la Sociedad Española de Medicina y Seguridad en el Trabajo (SEMST).

\section{Metodología}

El desarrollo de este documento se realizó en varias etapas, en las que la evidencia disponible se revisó en ciclos de análisis y discusión. El proceso tuvo lugar de la siguiente manera:

1. Cuatro miembros de la AEDV desarrollaron un primer borrador del documento que incluía información actualizada sobre la definición, la clasificación, el diagnóstico y el tratamiento del EM. La búsqueda bibliográfica se realizó utilizando la base de datos Medline (US National Library of Medicine ${ }^{\circledR}$ ) centrándose en la literatura de los últimos 10 años (2008-2018), y los resultados se filtraron según la relevancia y el interés relacionado con los objetivos de esta guía, aunque no se usaron los criterios PRISMA de revisiones sistemáticas. Se usaron combinaciones de palabras clave que sistemáticamente tomaban en cuenta todos los posibles usos relacionados («dermatitis de manos», «eccema de manos», «dermatitis palmar») y calificadores («epidemiología», «prevención», «etiología», «clasificación», «fisiopatología», «tratamiento»). También se llevaron a cabo búsquedas completas de denominaciones de fármacos específicos (p.ej., «inhibidores de la calcineurina», «alitretinoína»). El desarrollo del borrador también consideró, entre otros aspectos, las guías previas de la Sociedad Europea de Dermatitis de Contacto (ESCD) para el manejo del eccema de manos ${ }^{3}$, el documento de posicionamiento de la acción de Cooperación Europea en Ciencia y Tecnología (COST) StanDerm (TD 1206) sobre enfermedades de la piel relacionadas con el trabajo y profesionales ${ }^{2}$, las guías canadienses de manejo del $E M^{4}$ o las directrices danesas del Grupo de Dermatitis de Contacto para el diagnóstico y tratamiento del $E M^{5}$.

2. El borrador fue discutido en detalle en mayo de 2018 entre el panel de la AEDV y los participantes de una reunión que incluyó un médico de atención de cada una de las sociedades (D.P.-M. por SEMERGEN; R.S.S. por semFYC; R.S.C. por SEMG y A.A.G. por SEMST) con el objetivo de asegurar que esta guía incluía todos los posibles puntos de vista de las alternativas de práctica clínica. 
3. Finalmente, se desarrolló un borrador final que luego se usó para crear un resumen de las pautas que utilizarán los médicos de AP y de medicina del trabajo en la práctica clínica diaria. Uno de los objetivos principales de este documento fue estandarizar los criterios de derivación de los pacientes con EM a especialistas. No obstante, esta guía no está formalmente avalada por las sociedades de médicos de AP (SEMERGEN, semFYC, SEMG, y SEMST) implicadas en su desarrollo.

\section{Definición}

El EM, o dermatitis de manos, es una enfermedad multifactorial que comprende diversas condiciones etiológicas y morfológicas. Se trata principalmente de un trastorno inflamatorio de la piel de las manos que es muy molesto y puede producir una gran afectación de la calidad de vida del paciente.

Las características clínicas del EM pueden incluir eritema, edema, vesículas, costras, descamación, liquenificación, hiperqueratosis y fisuración. El EM puede considerarse agudo si predominan las vesículas y costras, o crónico si predomina la hiperqueratosis. El EM requiere una intervención temprana porque tiene tendencia a convertirse en crónico $0^{6}$. Si el proceso eccematoso se prolonga de forma continua 3 meses o más, o si recae dos veces o más en un año, a pesar de las medidas terapéuticas adecuadas y buena adherencia terapéutica, se trata de un $\mathrm{ECM}^{3}$.

\section{Epidemiología}

Existen numerosos estudios epidemiológicos que describen la prevalencia del EM en la población general y en profesiones específicas ${ }^{1,7}$. Una gran revisión de estudios de 1964 a 2007 reveló que la prevalencia puntual del EM en la población general era de alrededor del 4\%, la prevalencia de 1 año cerca del $10 \%$ y la prevalencia de vida del $15 \%{ }^{1}$. Se han encontrado valores bastante similares en estudios recientes que han incluido un gran número de pacientes, especialmente en países escandinavos. Por ejemplo, un gran estudio epidemiológico en Noruega ( $>50.000$ personas) reveló que la prevalencia del EM en la población general era del 11,3\%, y que en el $4,8 \%$ de la población estaba relacionado con el trabajo ${ }^{8}$. Un estudio de prevalencia en adolescentes suecos mostró que la prevalencia del EM a 1 año era del $5,2 \%$ y la prevalencia a lo largo de la vida era del $9,7 \%$, y que esta incidencia era de la misma magnitud en adultos ${ }^{9}$. Otro estudio de adultos suecos mostró que la prevalencia de 1 año fue del $15,8 \%$ (el doble en mujeres que en hombres) ${ }^{10}$. Un estudio danés mostró que la prevalencia del EM a 1 año en adultos de $28-30$ años fue del $14,3 \%{ }^{11}$.

En cuanto a la gravedad de la enfermedad, un estudio de la población italiana con EM reveló que el EM era crónico en el $83,5 \%$ de los pacientes; el $21,3 \%$ tenían eccema grave, y el $62,0 \%$ eran refractarios al tratamiento estándar ${ }^{12}$. Del registro CARPE de pacientes alemanes con ECM, el ECM fue muy grave en el $23,4 \%$, grave en el $47,0 \%$, moderado en el $20,1 \%$ y leve o muy leve en el $9,6 \%$ de los pacientes ${ }^{13}$. De un estudio de adolescentes suecos con EM, el 27,0\% tenían EM de moderado a grave ${ }^{9}$. Sin embargo, el EM suele ser una enfermedad con curso remitente y recurrente, y el grado de gravedad puede cambiar rápidamente en cada paciente individual.

\section{Factores de riesgo}

Los factores de riesgo asociados al EM son muy variados, especialmente cuando están asociados a la alergia. Sin embargo, algunos factores generales han sido consistentemente identificados como riesgos para el desarrollo de EM:

- Dermatitis atópica ${ }^{1,10,11,14-20}$. Una historia de dermatitis atópica es el predictor más fuerte de ECM en niños y adultos. La dermatitis atópica es la causa más frecuente de $E M$ en niños ${ }^{20}$. Un estudio de $E M$ en la población general danesa mostró que las mutaciones de filagrina están asociadas con ECM en pacientes con dermatitis atópica ${ }^{16}$.

- Otras enfermedades atópicas, como el asma y la rinitis alérgica ${ }^{14}$.

- Hábito tabáquico ${ }^{21-25}$. Un metaanálisis reciente no mostró que el tabaquismo sea un factor de riesgo para el EM, como se había sugerido, pero no se puede excluir una asociación que indique que fumar puede influir en el curso del $\mathrm{EM}^{26}$. Un estudio reciente de pacientes de $\mathrm{EM}$ relacionados con el trabajo de Dinamarca encontró una fuerte asociación entre el tabaquismo y la gravedad del $E M^{21}$. Es evidente que se necesitan más estudios, pero es importante considerar el abandono del hábito de fumar como parte del tratamiento del $\mathrm{EM}^{23}$.

- Piel seca ${ }^{14}$.

- Trabajos con humedad, lavado excesivo de las manos $111,14,18,27-29$. Como el EM puede tener implicaciones a nivel profesional y puede ser considerado como una enfermedad profesional, su impacto social y económico es significativo. Los trabajadores con mayor riesgo eran los que realizaban el procesamiento de alimentos y trabajos que implicaban el constante contacto con agua, como los profesionales de la salud - la peluquería, entre otros ${ }^{12}$. Los trabajadores de la salud han sido detectados como un grupo de alto riesgo en estudios realizados en $\mathrm{Canadá}^{30}$, Corea ${ }^{18}$, Países Bajos $^{31}$, Dinamarca ${ }^{19,28,32}$, Noruega ${ }^{8}$ y Alemania $^{15}$, con prevalencias en este grupo que oscilan entre el 21,0 y el $47,0 \%$. Los trabajadores de la salud en Dinamarca con EM presentaron pruebas epicutáneas positivas en el $53 \%$ de los casos, principalmente al níquel, tiomersal, fragancias, productos químicos de goma y colofonia ${ }^{32}$. Un estudio epidemiológico en enfermeras alemanas con enfermedades de la piel de origen laboral mostró que el $90,3 \%$ sufrían de $E M$, y el $13,5 \%$ de estas tenían colonización concomitante por Staphylococcus aureus resistente a la meticilina (SARM), principalmente en la nariz $(81,4 \%)$. De estos, aproximadamente el $55,8 \%$ también tuvieron las manos afectadas ${ }^{15}$. Los odontólogos en Japón también tienen una alta prevalencia $(46,4 \%)$ de $\mathrm{ECM}^{14}$. En un estudio reciente, los peluqueros informaron altas tasas de incidencia de EM en comparación con la población general, lo que a menudo condujo a la interrupción del trabajo ${ }^{33}$. Los peluqueros presentaron altas tasas de EM en estudios en Dinamarca ${ }^{34,35}$, Turquía ${ }^{36}$ y $\mathrm{Croacia}^{37}$. En otras regiones del mundo la situación puede ser sustancialmente diferente. Por ejemplo, un 
estudio en la India mostró que los agricultores, los trabajadores de la construcción y las amas de casa sufren la mayor parte de la dermatitis de contacto profesional, de los cuales el $81,2 \%$ es $E M^{38}$. Los alérgenos más frecuentes fueron el Parthenium hysterophorus en los agricultores, el dicromato de potasio en los trabajadores de la construcción y las verduras en las amas de casa.

- Varios estudios han revelado que el EM suele ser más común en mujeres que en hombres, pero se desconoce la razón precisa de este efecto ${ }^{1,8,10,11,39,40}$.

- Presencia de ciertas mutaciones en el gen de la filagrina (ver la sección siguiente).

\section{Fisiopatología}

El EM es un grupo de condiciones muy heterogéneo y, por lo tanto, no existen mecanismos patológicos definidos que puedan aplicarse de manera general. Sin embargo, se acepta que factores genéticos, como por ejemplo las mutaciones del gen de la filagrina (FLG), y factores externos, como el contacto con irritantes cutáneos, alteran la estructura y la composición del estrato córneo provocando que la función barrera cutánea sea defectuosa y favoreciendo el EM. Las mutaciones de FLG se han asociado con la presencia de EM, especialmente en las variantes endógenas ${ }^{41}$.

La barrera cutánea defectuosa desempeña un papel en la patogénesis del ECM y puede conducir tanto a un EM de tipo irritativo como alérgico ${ }^{42}$. En un estudio reciente se encontró que algunas proteínas importantes de barrera cutánea, como la filagrina, la hornerina, las peptidasas KLK5 y KLK7 y la cistatina $E / M$, se regulan negativamente en el EM ${ }^{42}$. La barrera cutánea se puede ver alterada bien por factores de origen genético o bien por causas exógenas. Los trabajos con humedad son una de las principales causas de irritación cutánea, y ello facilita la sensibilización a antígenos en el $\mathrm{EM}^{43}$.

El papel que el microbioma tiene en el desarrollo del ECM ha adquirido interés recientemente. La tasa de colonización por S. aureus es superior en las manos de pacientes con EM en comparación con individuos sanos, mostrando los pacientes con EM grave una densidad de S. aureus significativamente mayor respecto a los pacientes con eccema más leve ${ }^{44}$.

Se ha propuesto que interleucinas del tipo IL-1 (IL-36 $\alpha$ ) podrían estar implicadas en la inflamación cutánea en el $\mathrm{ECM}^{45}$. Por esta razón, la IL-36 $\alpha$ podría ser un candidato a biomarcador que podría ayudar en el complejo proceso de diagnóstico de $\mathrm{ECM}^{45}$.

\section{Clasificación}

La identificación adecuada del tipo de EM es el primer paso para implementar tratamientos efectivos y eficientes ${ }^{12}$. Además, en estudios epidemiológicos y clínicos la clasificación del EM se hace imperativa para estratificar a los pacientes. Se han propuesto varias clasificaciones para los tipos EM basados en la morfología clínica y la etiología. Sin embargo, no existe una relación simple entre ellas, ya que los diferentes tipos de EM pueden presentarse y progresar con características distintas en cada individuo. Actualmente no hay una clasificación universalmente aceptada del EM.
Tabla 1 Tipos de eccema de manos (EM)

EM de contacto irritativo

Variado, pero frecuentemente con fisuras o pulpitis Normalmente afecta espacios interdigitales Causado por exposición prolongada o repetida a irritantes, lavado frecuente de manos, uso de guantes oclusivos, sustancias alcalinas o aceites

EM de contacto alérgico

Afecta al sitio de contacto (palmas o las puntas de los dedos), aunque con preferencia el dorso de las manos o dedos, donde la piel es más vulnerable

Causado por exposición a alérgenos

Suelen identificarse bien las fases de eritema/vesícula y posterior escoriación, rascado y liquenificación

EM de contacto por proteínas

Inicialmente con una fase de lesiones habonosas o eccematosas

Causado por alérgenos alimentarios, látex o materiales de origen biológico

Dermatitis atópica

Con frecuencia afectación de muñecas, con picor Dermatitis atópica previa, asma o rinitis

EM vesicular

Recurrente con erupción vesicular en palmas de las manos

Puede afectar lados de los dedos

Causado por estrés, dermatitis de contacto sistémica, alérgenos al polvo o infecciones fúngicas

EM hiperqueratósico

Zonas hiperqueratósicas claramente demarcadas en palmas de la mano, sin vesículas o pústulas Algunos autores solo consideran eccema hiperqueratósico aquel que previamente ha sido vesicular

Según el Grupo Danés de Dermatitis de Contacto, existen seis tipos clínicos (morfológicos) de EM: EM vesicular recurrente, EM fisurado crónico, eccema palmar hiperqueratósico, pulpitis, eccema interdigital y EM numular ${ }^{5,6,46,47}$.

En la tabla 1 se muestran los tipos comunes de $\mathrm{EM}^{3,6,48,49}$. Según un estudio reciente, hasta un $89 \%$ de los pacientes podrían clasificarse según estos subgrupos de diagnóstico, y otro $7 \%$ cuando utilizan dos diagnósticos principales ${ }^{50}$. Sin embargo, algunos autores estiman que al menos el $20-26 \%$ de los casos de ECM no encajan en ninguno de estos grupos etiológicos y podrían considerarse idiopáticos ${ }^{5,49}$. Las formas mixtas de ECM pueden ocurrir con frecuencia6,50. Por ejemplo, un estudio retrospectivo de pacientes daneses de dermatitis de contacto laboral mostró que el 6,4\% tenían un diagnóstico combinado de dermatitis de contacto alérgica e irritativa ${ }^{51}$. También la dermatitis de contacto puede ser concurrente con la dermatitis atópica ${ }^{52-54}$.

\section{Diagnóstico}

El diagnóstico de EM es clínico y se basa en la anamnesis y la exploración física. Las pruebas diagnósticas, entre las que se encuentran las pruebas epicutáneas (patch test), las pruebas de punción (prick test), los test microbiológicos y las 
Tabla 2 Anamnesis de un paciente con posible eccema de manos (EM)

- Historial personal y familiar de alergias y enfermedades cutáneas

- Historial laboral

- Patrón de EM (agudo, crónico, recurrente), con descripción de exacerbaciones y remisiones con respecto a trabajo (vacaciones, fines de semana), estaciones o hábitos de vida

- Exposiciones a irritantes o alérgenos (tipo, tiempo de exposición, frecuencia) en el trabajo o en casa del paciente

- Hábitos en lavado de manos, cuidado de la piel o uso de guantes

- Efectos en calidad de vida (relaciones sociales, trabajo, vida familiar)

Tabla 3 Diagnóstico diferencial

Psoriasis
Historia familiar de psoriasis
Hiperqueratosis delimitada, en placas discoideas,
palmar
Afectación del cuero cabelludo, codos, rodillas, nalgas
Enfermedad de larga trayectoria y refractaria a
tratamiento
Tiña manum
Típicamente unilateral
Afecta a los pliegues palmares predominantemente
Onicomicosis o tiña pedis asociadas

biopsias cutáneas, son útiles para descartar otras patologías o para intentar establecer un diagnóstico etiológico ${ }^{3,49}$.

El primer paso en el diagnóstico del EM es la anamnesis. Una lista de los principales aspectos a tratar durante la entrevista se muestra en la tabla 2 , donde tanto el historial personal como el laboral son elementos esenciales que deben considerarse. Los hallazgos más significativos deberían quedar siempre reflejados en el historial clínico del paciente.

La inspección física debería incluir toda la piel, y especialmente pies, así como la búsqueda de síntomas clínicos de dermatofitosis. La diferenciación clínica entre ECM y otras enfermedades de la piel de la mano puede ser difícil, especialmente con psoriasis y micosis ${ }^{6,54}$. En la tabla 3 se muestran algunas claves del diagnóstico diferencial con otras patologías dermatológicas. La psoriasis se caracteriza por una hiperqueratosis claramente delimitada en placas discoideas y ausencia de picazón o vesículas, así como la presencia de lesiones en cuero cabelludo, uñas, zonas de extensión de codos y rodillas, y nalgas. Además, los pacientes típicamente tienen una historia personal o familiar de psoriasis o dermatitis seborreica persistente. Las infecciones micóticas de la piel, aparte de la inspección de signos clínicos, se diagnostican con un ensayo de hidróxido de potasio $(\mathrm{KOH})$ y el cultivo directo de hongos. Otras afecciones que pueden confundirse con ECM incluyen el liquen plano, la sarna, el granuloma anular, el herpes simple, el eritema multiforme, la pitiriasis rubra pilaris y la dermatomiositis ${ }^{6,54}$.
Tabla 4 Pruebas clínicas usadas en el diagnóstico del eccema crónico de manos (ECM)

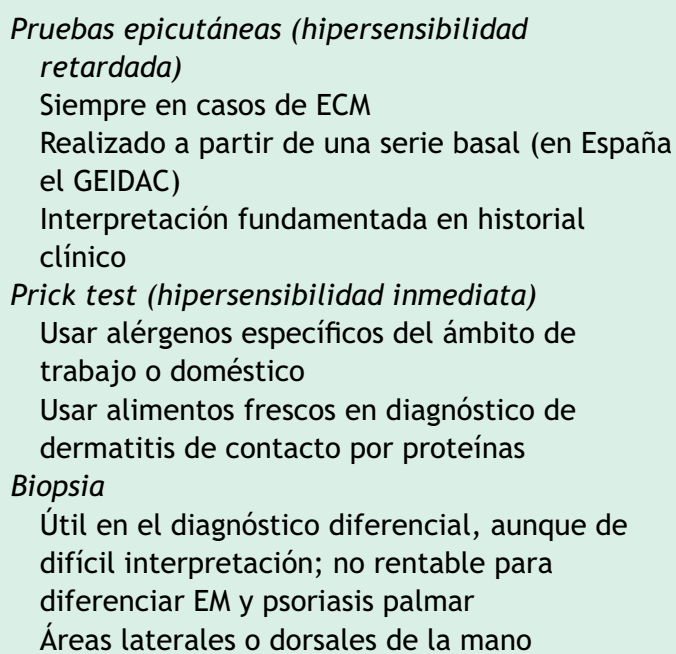

En la tabla 4 se resumen las herramientas disponibles en el ámbito clínico, donde la prueba diagnóstica por excelencia es la prueba del parche o epicutánea. La realización de pruebas epicutáneas ha de considerarse en cualquier ECM, independiente de la morfología de este o de la presunción diagnóstica. Además, las pruebas epicutáneas han de ser consideradas siempre en pacientes con EM resistente a la terapia, EM recurrente, EM con distribución atípica/cambiante o patrones sugestivos de EM alérgico de contacto ${ }^{55}$. Tanto las pruebas epicutáneas como los prick test sirven para descartar procesos alérgicos como la causa del EM. Típicamente la biopsia cutánea y el cultivo micológico son herramientas útiles solo en el diagnóstico diferencial con otras patologías cutáneas. El EM puede ser inducido por una amplia gama de factores externos e internos que actúan solos o en combinación, y este hecho debe tenerse en cuenta al hacer un diagnóstico.

La figura 1 muestra un algoritmo para el diagnóstico etiológico del ECM. El ECM de contacto alérgico tiene como herramienta diagnóstica esencial las pruebas epicutáneas ${ }^{56}$. El ECM de contacto irritativo carece de pruebas diagnósticas de provocación aplicables en la práctica clínica habitual útiles para evaluar las reacciones a los irritantes. Por ello el diagnóstico a menudo se basa en el patrón clínico, el análisis de los productos manipulados y la ausencia de reacciones de pruebas epicutáneas positivas (lo que indicaría una alergia de contacto). Sin embargo, en muchas ocasiones el EM de contacto irritativo y el alérgico con frecuencia ocurren juntos ${ }^{51,56}$.

El EM de contacto por proteínas se sospecha clínicamente por la aparición inmediata de prurito, habones o eccema tras el contacto con material biológico, como fruta, pescado, vísceras, etc. Es decir, el contacto continuado con proteínas es responsable del EM, y por ello la prueba diagnóstica utilizada en el diagnóstico del EM de contacto proteínico es el prick test y no las pruebas epicutáneas (que detectan alergia a haptenos). En muchas ocasiones se precisará la realización de prick-prick sobre los alimentos sospechosos aportados por el propio paciente. Si bien los agentes responsables del EM alérgico clásico son agentes químicos de un peso 


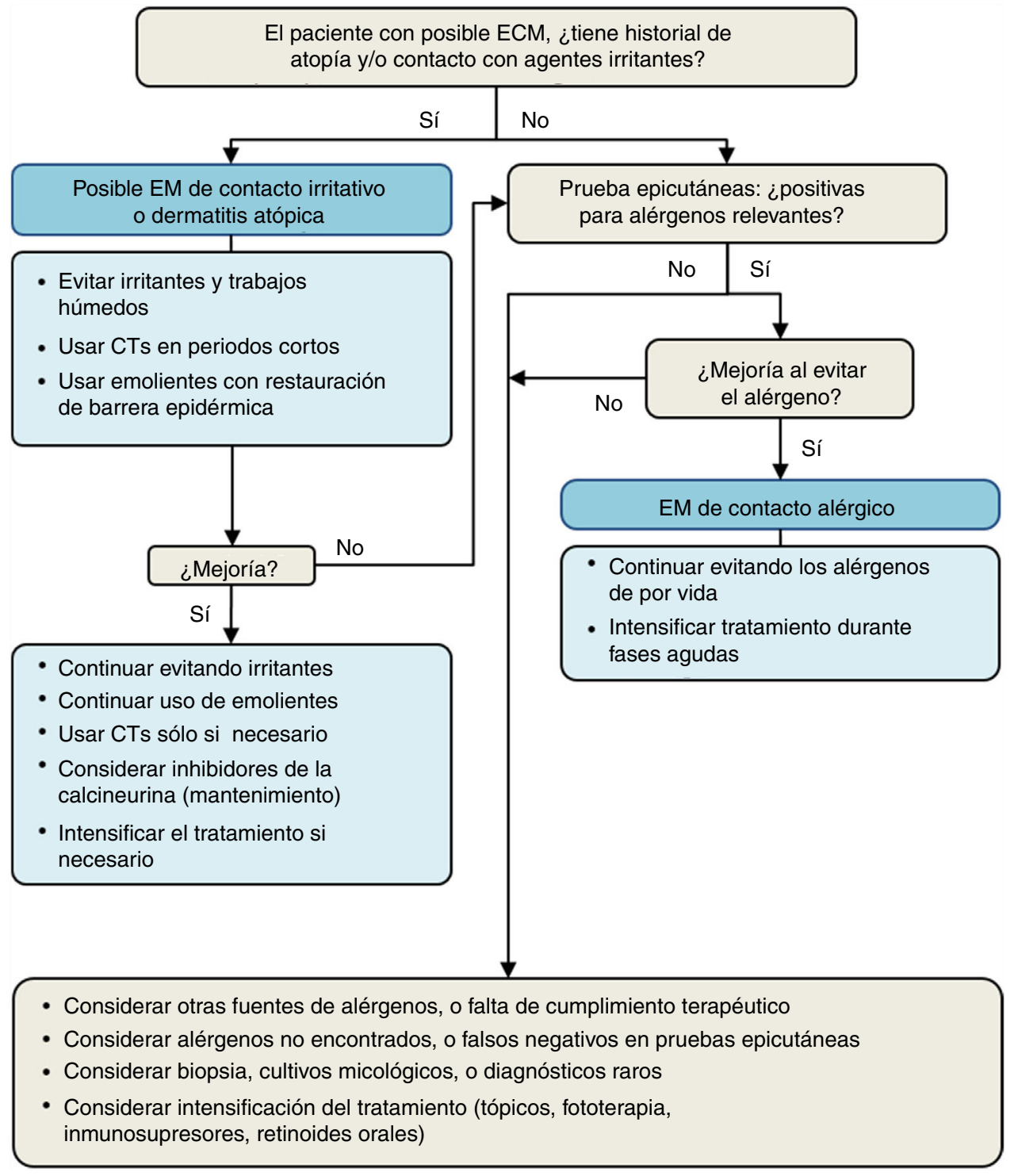

Figura 1 Diagnóstico etiológico del eccema de manos (EM).

molecular pequeño, en el que el EM de contacto está inducido por proteínas, las pruebas epicutáneas clásicas suelen ser negativas. Este tipo de EM a menudo ocurre en pacientes con una barrera dérmica-epidérmica dañada debido a la atopia o a la alteración previa por irritantes.

\section{Prevención}

Las medidas para mitigar o disminuir la incidencia del EM se pueden clasificar como primarias, secundarias y terciarias, según la etapa de desarrollo de la enfermedad. Así, la prevención primaria tiene como objetivo disminuir la incidencia del EM en la población sana, la prevención secundaria aborda las medidas a tomar cuando se está desarrollando el EM y evitar que se convierta en crónico, y la prevención terciaria guía los pasos a seguir en pacientes con ECM. Un resumen de las medidas de prevención en los tres niveles se puede encontrar en la tabla 5.

\section{Prevención primaria}

La prevención primaria es fundamental y debe comenzar en las escuelas, especialmente a los niños con enfermedades atópicas, ya que tienen un mayor riesgo de desarrollar EM más adelante en su la vida. En prevención primaria el objetivo es evitar la exposición al agente nocivo, potencialmente responsable de enfermedad e identificado como un riesgo para la población.

Un paso crucial en el proceso de prevención primaria es la evaluación de riesgos laborales, que se basa en la identificación y cuantificación de estos riesgos en el lugar de trabajo. Esto es seguido por su clasificación y esfuerzos multidisciplinarios para definir las acciones preventivas más apropiadas ${ }^{2}$. Las medidas de prevención 
Tabla 5 Prevención del eccema de manos (EM)

\begin{tabular}{|c|c|}
\hline $\begin{array}{l}\text { Nivel de } \\
\text { prevención }\end{array}$ & Posibles medidas preventivas \\
\hline Primaria & $\begin{array}{l}\text { Uso de guantes y humectantes para } \\
\text { manos (tablas } 6 \text { y } 7 \text { ) } \\
\text { Evitación de exposición a través de } \\
\text { cambios en la legislación de } \\
\text { materiales permitidos (p.ej., } \\
\text { presencia de ciertos conservantes en } \\
\text { cosméticos o de cromo en cementos) } \\
\text { Sistemas de ventilación que reduzcan } \\
\text { la exposición a alérgenos o } \\
\text { materiales irritantes } \\
\text { Vigilancia de la salud específica de } \\
\text { trabajadores para identificar } \\
\text { individuos sensibilizados antes de que } \\
\text { los primeros síntomas aparezcan } \\
\text { Educación general sobre el uso de } \\
\text { guantes, productos para el cuidado } \\
\text { de la piel y otras medidas de } \\
\text { protección }\end{array}$ \\
\hline Secundaria & $\begin{array}{l}\text { Exámenes regulares en el lugar de } \\
\text { trabajo para identificar y tratar } \\
\text { síntomas tempranos de EM } \\
\text { Uso de humectantes y otras medidas } \\
\text { preventivas tópicas del cuidado de la } \\
\text { piel, dirigido por dermatólogos } \\
\text { Educación en el lugar de trabajo por } \\
\text { parte de dermatólogos bajo } \\
\text { programas específicos sobre el } \\
\text { cuidado de la piel (seminarios, } \\
\text { folletos, cursillos especializados) }\end{array}$ \\
\hline Terciaria & $\begin{array}{l}\text { Los pacientes deberían considerar el } \\
\text { abandono temporal del trabajo para } \\
\text { evitar la sensibilización y recobrar } \\
\text { plenamente las funciones de la } \\
\text { barrera epidérmica } \\
\text { Enfoque concertado e } \\
\text { interdisciplinario a pacientes con } \\
\text { ECM y que implique a dermatólogos, } \\
\text { especialistas en medicina del trabajo } \\
\text { y otros profesionales }\end{array}$ \\
\hline
\end{tabular}

primaria se dividen en cuatro áreas principales: medidas técnicas/organizativas, protección personal (higiene de manos, uso de guantes y humectantes), programas educativos y acceso a especialistas ${ }^{2}$.

Entre las medidas organizativas, una estrategia efectiva ha sido la introducción de legislación para reducir o eliminar ciertos alérgenos (p.ej., cromato en trabajadores de la construcción, monotioglicolato de glicerilo entre peluqueros $)^{57,58}$. El uso de guantes médicos sin aceleradores puede ser una alternativa efectiva en trabajadores de la salud que son alérgicos a los aceleradores de caucho ${ }^{59}$.

La principal herramienta de protección primaria en el EM es el uso de guantes de protección (tabla 6). Los pacientes siempre deben usar guantes en manos secas y limpias para trabajos con humedad o trabajos con sustancias peligrosas, tantas veces como sea necesario pero durante el
Tabla 6 Medidas generales: uso de guantes

- Usar guantes cuando se realizan trabajos con humedad

- No se deben usar guantes por largos periodos de tiempo. Si se emplean $>15 \mathrm{~min}$, usar guantes de algodón debajo

- Si utiliza guantes durante toda su jornada laboral es aconsejable tener tres pares de guantes de algodón para ir cambiándolos. Una vez el guante de algodón está húmedo pierde su papel protector

- Los guantes deben estar intactos, limpios y secos por dentro

- La elección del tipo de guante dependerá del tipo de material que se está manipulando: guantes de algodón para cartón o papel; guantes de serraje o cuero si se manejan sólidos; guantes de plástico desechables si se manejan alimentos; guantes de vinilo, nitrilo o goma si se manejan líquidos

menor tiempo posible ${ }^{2}$. Los guantes de un solo uso deben usarse solo una vez y nunca deben reciclarse, y los guantes defectuosos deben retirarse inmediatamente. Con respecto al tipo de guantes, el algodón debe usarse para el trabajo doméstico general si es un trabajo en seco (como la limpieza del polvo), y se deben usar guantes de algodón por debajo de guantes de otros materiales si estos se usan por más de $15 \mathrm{~min}^{2}$. Los guantes de látex protegen bien contra microorganismos o materiales acuosos, pero ofrecen poca protección a aceites, solventes y productos químicos. Los guantes de nitrilo son protectores contra aceites y solventes, y los guantes de vinilo ofrecen protección contra la mayoría de los productos químicos ${ }^{49}$.

Un estudio en los Países Bajos abogó por una estrategia de implementación múltiple que consistía en educación enfocada en grupos de trabajo, recordatorios en el lugar de trabajo y folletos, con buenos resultados generales tras una evaluación de 6 meses $^{60,61}$. Sin embargo, esta metodología

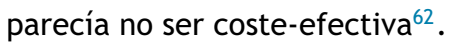

\section{Prevención secundaria}

El objetivo de la prevención secundaria debe ser la detección temprana, el diagnóstico y el tratamiento de la enfermedad por parte de especialistas. La educación del paciente debe inducir cambios de comportamiento en el trabajo o en el hogar, protección de la piel y eliminación de alérgenos o sustancias irritantes. Se ha sugerido que la información debe darse en formato escrito ${ }^{49,63}$. Además, el asesoramiento laboral es un enfoque eficaz para alentar la prevención secundaria en adultos jóvenes en riesgo, como aquellos con dermatitis atópica, que inician ocupaciones de alto riesgo, según un estudio en Alemania ${ }^{64}$. Un estudio alemán ha demostrado la efectividad a largo plazo de la prevención secundaria interdisciplinaria, señalando que la detección temprana y la notificación de las etapas iniciales de la enfermedad son de suma importancia ${ }^{65}$.

El uso de cremas protectoras y emolientes es crítico en esta etapa, ya que pueden ser instrumentos efectivos 
Tabla 7 Medidas generales: uso de emolientes

- Se pueden emplear en todas las fases del tratamiento y en todo tipo de eccema, salvo en aquellos muy agudos o muy fisurados, pues su absorción puede favorecer la sensibilización frente alguno de sus componentes

- En eccema de manos (EM) descamativo/hiperqueratósico, el emoliente debe ser graso; en EM exudativo el emoliente debe ser acuoso

- Durante el día es preferible el uso de lociones y cremas, y por la noche, pomadas

para prevenir la progresión de la enfermedad (tabla 7). Las cremas barrera están diseñadas para crear un escudo protector en la piel y solo deben usarse en pieles sanas (y no en inflamadas). Protegen contra irritantes comunes como agua, detergentes, metales, resinas, materiales oleosos o luz ultravioleta, y pueden ayudar en la higiene de la piel $^{49}$. Es importante se señalar, no obstante, que las cremas barrera pueden dar una falsa impresión de protección que haga que el paciente se exponga de manera indebida a alérgenos e irritantes. Las cremas barrera en ningún caso sustituyen a las medidas físicas, como el uso de guantes. Por otro lado, existe una gran variedad de emolientes que pueden recomendarse y usarse clínicamente, y es ampliamente aceptado que pueden mejorar la hidratación, prevenir el picor y ayudar a reparar la barrera cutánea. La aplicación de humectantes podría prolongar el intervalo libre de enfermedad en pacientes con EM controlado ${ }^{66}$. Sin embargo, la evidencia es limitada en lo concerniente a la eficacia clínica de cualquier formulación específica y, en general, no se pueden hacer recomendaciones definitivas ${ }^{3}$. No se recomienda su uso durante las horas de trabajo debido a un mayor riesgo de sensibilización con los alérgenos causantes del EM, sino profilácticamente después de las horas de trabajo para facilitar la regeneración de la barrera cutánea. Actualmente se están desarrollando «dispositivos emolientes recetados» con mezclas complejas que pretenden imitar los componentes de la piel para tratar lesiones específicas ${ }^{67}$.

\section{Prevención terciaria}

La prevención terciaria se aplica a los pacientes con EM que no han podido prevenir el desarrollo de enfermedades crónicas. Los principales objetivos son, por tanto, la reducción de la gravedad del EM, la reducción del uso de corticosteroides y la facilitación del retorno al trabajo y la mejora de la calidad de vida. En estos casos es aconsejable la interrupción temporal del trabajo para evitar la sensibilización si el EM es de etiología irritativa, y favorecer la recuperación completa de la barrera cutánea. El enfoque recomendado es siempre interdisciplinario e involucra a dermatólogos y especialistas en medicina del trabajo ${ }^{3}$. El «modelo Osnabrück» se ha sugerido como una vía eficaz a largo plazo para la rehabilitación de pacientes ${ }^{68,69}$. En una evaluación reciente de los efectos a largo plazo de un programa de prevención individualizado, el $96,9 \%$ de los pacientes pudieron volver a trabajar nuevamente, el $82,7 \%$

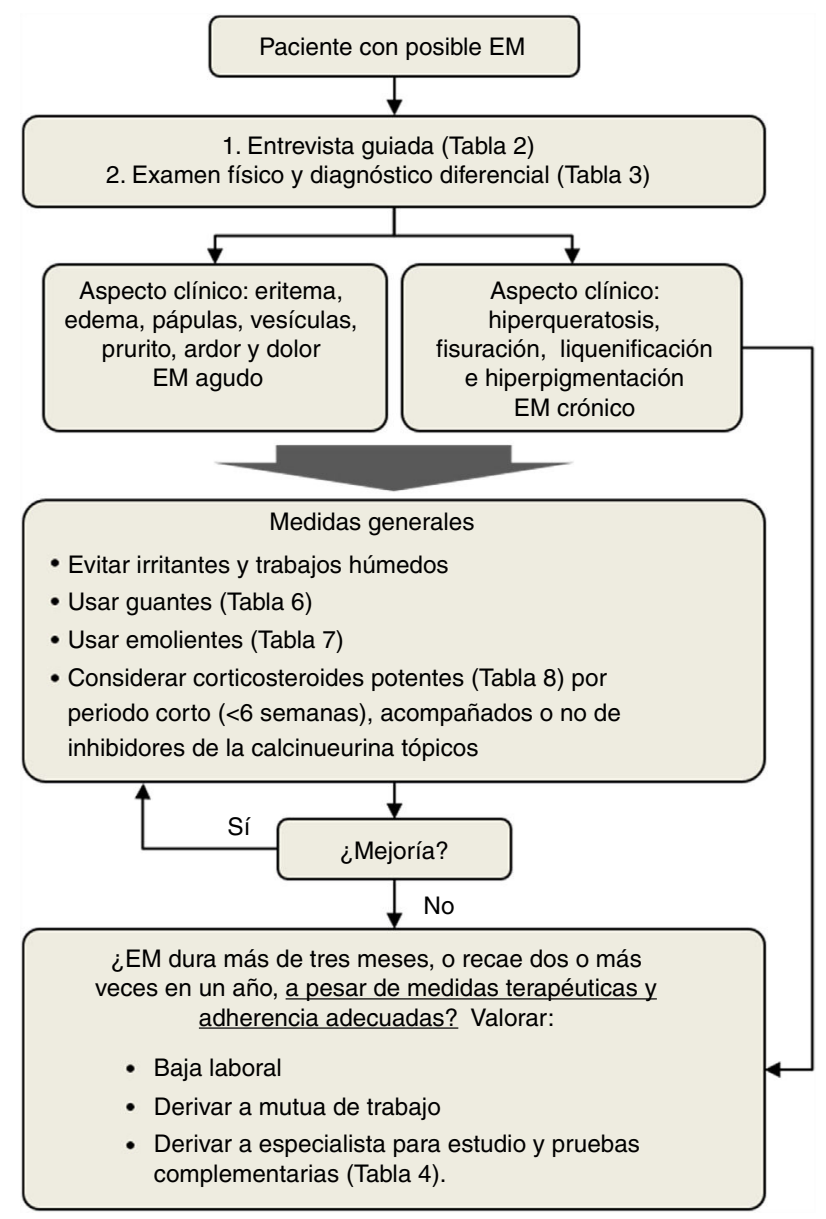

Figura 2 Manejo del eccema de manos (EM) por el médico de atención primaria.

Tabla 8 Uso de corticosteroides

Los corticosteroides comúnmente utilizados son de alta potencia: furoato de mometasona al $0,1 \%$ en crema; valerato de betametosona al 0,05 o al $0,1 \%$ en crema

Los corticosteroides tópicos se deben usar por periodos cortos (<6 semanas)

Considerar corticoides orales en caso de exacerbaciones si el eccema es muy invalidante y no se controla con otras medidas. Pauta descendente desde $0,5-1 \mathrm{mg} / \mathrm{kg} /$ día, $1-2$ semanas

seguían trabajando después de 3 años y el 75,0\% no habían cambiado de profesión ${ }^{68}$.

\section{Tratamiento}

El tratamiento del EM debe personalizarse y adaptarse a variables como la edad, la historia clínica y la ocupación. Las recomendaciones terapéuticas deben tener en cuenta la etiología, la morfología y la ubicación de las lesiones cutáneas. En la figura 2 se muestra una guía del manejo de pacientes con posible EM por el médico de AP. El primer paso es la anamnesis y el examen físico completo, que guiará al 
médico en el diagnóstico diferencial y el tratamiento inicial. Las medidas generales se basan en la evitación de irritantes y trabajos con humedad, así como el uso de guantes y de emolientes (tablas 6 y 7 ). Si fuera necesario, se pueden considerar los corticosteroides tópicos de alta potencia (tabla 8). Los casos de ECM siempre han de derivarse al dermatólogo para realizar las pruebas complementarias necesarias y establecer un diagnóstico etiológico definitivo. Además, el médico de AP debería valorar la baja laboral para que pueda haber una recuperación efectiva.

En la tabla 9 se presenta un resumen de los tratamientos del EM y en la figura 3 se presenta un algoritmo de tratamiento por el especialista. El seguimiento personalizado es esencial porque la efectividad del tratamiento con frecuencia es difícil de estimar a largo plazo. Ello es debido a la falta de ensayos controlados aleatorizados definitivos que evalúen y comparen la efectividad de los tratamientos. Además, las comparaciones entre tratamientos a menudo son imposibles porque los diversos estudios clínicos reclutan poblaciones de pacientes diferentes y faltan medidas de resultados estandarizadas.

Es muy recomendable que el EM agudo se aborde terapéuticamente tan pronto como sea posible para prevenir el desarrollo de formas crónicas, cuyo tratamiento puede ser mucho más difícil. También es fundamental, cuando se conoce, evitar la reexposición de la piel a cualquier irritante o alérgeno, y permitir la regeneración de la barrera cutánea. Por esta razón, uno de los primeros objetivos de terapia para todos los pacientes debería ser identificar los factores exógenos causales, el establecimiento de cambios en el estilo de vida y medidas preventivas ${ }^{3}$.

\section{Tratamientos tópicos}

La mayoría de los casos de EM agudo se pueden manejar adecuadamente con una combinación de medidas preventivas y tratamiento tópico. Los tratamientos tópicos incluyen esteroides, inhibidores de la calcineurina (tacrolimus, pimecrolimus) y otros tratamientos tópicos (iontoforesis, alquitrán y soluciones astringentes y antisépticas, como el permanganato potásico y el acetato de aluminio) ${ }^{3}$.

\section{Corticosteroides tópicos}

Los corticosteroides tópicos (CT) son el tratamiento de primera línea para el EM y pueden ser altamente efectivos a corto plazo (tabla 5) 3,49,63. Sin embargo, se considera que inhiben la regeneración del estrato córneo y que a largo plazo pueden causar atrofia de la piel (tabla 8). El tratamiento durante más de 6 semanas debe ser excepcional y solo llevarse a cabo bajo supervisión médica ${ }^{3}$. Además, se deben considerar las reacciones de hipersensibilidad inmediatas y diferidas cuando se usan CT, que deben ser estudiadas mediante pruebas epicutáneas ${ }^{70}$. Por lo general, para tratar el EM se eligen los CT de alta potencia (valerato de betametasona al 0,05\% o al 0,1\% en crema, furoato de mometasona al $0,1 \%$ en crema) y la administración una vez al día es suficiente. Un ensayo clínico de 44 pacientes mostró que el tratamiento una vez al día con una crema de corticosteroides potentes (valerato de betametasona al $0,1 \%$ ) fue superior a dos veces al día, especialmente en el grupo de pacientes con EM moderado $^{71}$. Un estudio reciente ha demostrado que aproximadamente el $50 \%$ de los casos de ECM son refractarios a esteroides $^{39}$.

\section{Inhibidores de la calcineurina}

En caso de fracaso del tratamiento con CT o de generación de reacciones alérgicas, se pueden utilizar inhibidores tópicos de la calcineurina (tacrolimus al 0,1\% en pomada en personas mayores de 16 años y al 0,03\% en niños menores de 16 años; pimecrolimus al 1\% en crema) en el tratamiento del EM. Un estudio comparativo mostró que el tratamiento con betametasona y tacrolimus tuvo un efecto positivo sobre la relación ceramida/colesterol y disminuyó la respuesta inflamatoria en comparación con un emoliente ${ }^{72}$. Se ha sugerido que los inhibidores de la calcineurina pueden ser útiles en el tratamiento del EM en combinación con CT en tratamientos a más largo plazo ${ }^{49}$.

\section{Fototerapia}

La fototerapia es una opción terapéutica estándar en casos de ECM refractario a CT, aunque la opinión general es que el tratamiento a largo plazo puede aumentar el riesgo de cáncer de piel $^{3}$. El tratamiento preferido es el PUVA (tópico), aunque los posibles efectos adversos son eritema y quemaduras en la piel.

Un estudio reciente mostró los efectos potencialmente beneficiosos del láser excímero de $308 \mathrm{~nm}$ para el tratamiento del ECM, ya que el $69 \%$ de los pacientes presentaron una reducción significativa de las puntuaciones en el Physician Global Assessment (PGA) y el $70 \%$ de la puntuación total de lesiones y síntomas ${ }^{73}$. En comparación con otras terapias ultravioletas, una ventaja del láser excímero es la menor dosis acumulada de radiación ultravioleta al dirigirse a áreas específicas ${ }^{73}$.

\section{Tratamientos sistémicos}

\section{Alitretinoína}

La alitretinoína (ácido retinoico 9-cis) es un fármaco inmunomodulador y antiinflamatorio aprobado para su uso en el tratamiento del EM grave y crónico que no responde o responde inadecuadamente a los $\mathrm{CT}^{3,49,74,75}$. Por lo tanto, la alitretinoína se recomienda como tratamiento de segunda línea (en relación con los $\mathrm{CT}$ ) en pacientes con $\mathrm{ECM}^{3}$.

Varios ensayos y análisis clínicos ahora apoyan firmemente la eficacia y la seguridad de la alitretinoína en el tratamiento del ECM severo y refractario ${ }^{76-78}$. Un gran ensayo clínico de fase 3 con pacientes con ECM severo refractario mostró que se lograron buenas respuestas a alitretinoína oral en hasta el $48 \%$ de los pacientes, en comparación con el $17 \%$ para placebo en 24 semanas $(p<0,001)^{79}$. Un segundo estudio de fase 3 de 596 pacientes con ECM grave mostró resultados similares, ya que la respuesta a la alitretinoína se observó en el $40 \%$ de los pacientes, frente al $15 \%$ del placebo $^{80}$.

Un estudio para analizar la efectividad de la alitretinoína en pacientes con ECM recurrente mostró que la tasa de respuesta fue del $80 \%$ en pacientes tratados en comparación con el $8 \%$ con placebo, lo que sugiere que el tratamiento intermitente con alitretinoína es adecuado para el manejo a largo plazo del $\mathrm{ECM}^{81}$. Un estudio observacional con 
Tabla 9 Tratamientos del eccema de manos (EM). Recomendaciones relevantes

\begin{tabular}{|c|c|}
\hline \multicolumn{2}{|l|}{ Tópicos } \\
\hline \multirow[t]{3}{*}{ Emolientes } & El uso durante las horas de trabajo puede incrementar la sensibilización \\
\hline & La adherencia al tratamiento es importante para un tratamiento óptimo \\
\hline & Puede requerir la supervisión de un médico o personal de enfermería \\
\hline \multirow[t]{5}{*}{$\begin{array}{l}\text { Corticosteroides } \\
\text { tópicos }\end{array}$} & $\begin{array}{l}\text { La potencia de los corticosteroides tópicos (CT) y el tiempo de tratamiento deben ser } \\
\text { considerados con cuidado }\end{array}$ \\
\hline & La morfología y el estado del EM deben ser considerados al escoger la formulación \\
\hline & Las infecciones fúngicas deben ser descartadas previamente \\
\hline & La alergia al CT debe ser considerada como un acontecimiento adverso potencial \\
\hline & $\begin{array}{l}\text { Los tratamientos que duran excepcionalmente }>6 \text { semanas requieren seguimiento } \\
\text { médico, ya que puede llevar a atrofia de la piel }\end{array}$ \\
\hline \multirow[t]{2}{*}{$\begin{array}{l}\text { Inhibidores de la } \\
\text { calcineurina }\end{array}$} & $\begin{array}{l}\text { Se pueden utilizar en combinación con CT para conseguir periodos más largos de } \\
\text { remisión del EM }\end{array}$ \\
\hline & Se pueden usar durante fases agudas de la enfermedad \\
\hline \multicolumn{2}{|r|}{ r } \\
\hline \multirow{2}{*}{$\begin{array}{l}\text { Corticosteroides } \\
\text { sistémicos }\end{array}$} & Solo para fases agudas de EM, no recomendados para eccema crónico de manos (ECM) \\
\hline & $\begin{array}{l}\text { El tratamiento no debe prolongarse más allá de } 3 \text { semanas debido a los potenciales } \\
\text { efectos adversos }\end{array}$ \\
\hline \multirow[t]{4}{*}{ Alitretinoína } & Aprobado su uso para ECM en caso de fallo a la respuesta de CT \\
\hline & El tratamiento puede mejorar sustancialmente el ECM en 12 semanas de tratamiento \\
\hline & Los efectos adversos son posibles (p.ej., dolor de cabeza, náuseas, hiperlipidemia) \\
\hline & Teratogénico, debe ser evitado por mujeres que quieran quedar embarazadas \\
\hline Acitretina & Efectivo, pero con un mayor índice de eventos adversos que la alitretinoína \\
\hline \multirow{2}{*}{ Ciclosporina } & Posible tratamiento cuando otras terapias fallan \\
\hline & Efectivo en dermatitis atópica \\
\hline \multirow[t]{2}{*}{ Azatioprina } & Posible tratamiento cuando otras terapias fallan \\
\hline & $\begin{array}{l}\text { Algunos pacientes acumulan metabolitos del medicamento que deben ser controlados } \\
\text { para evitar hepatotoxicidad }\end{array}$ \\
\hline
\end{tabular}
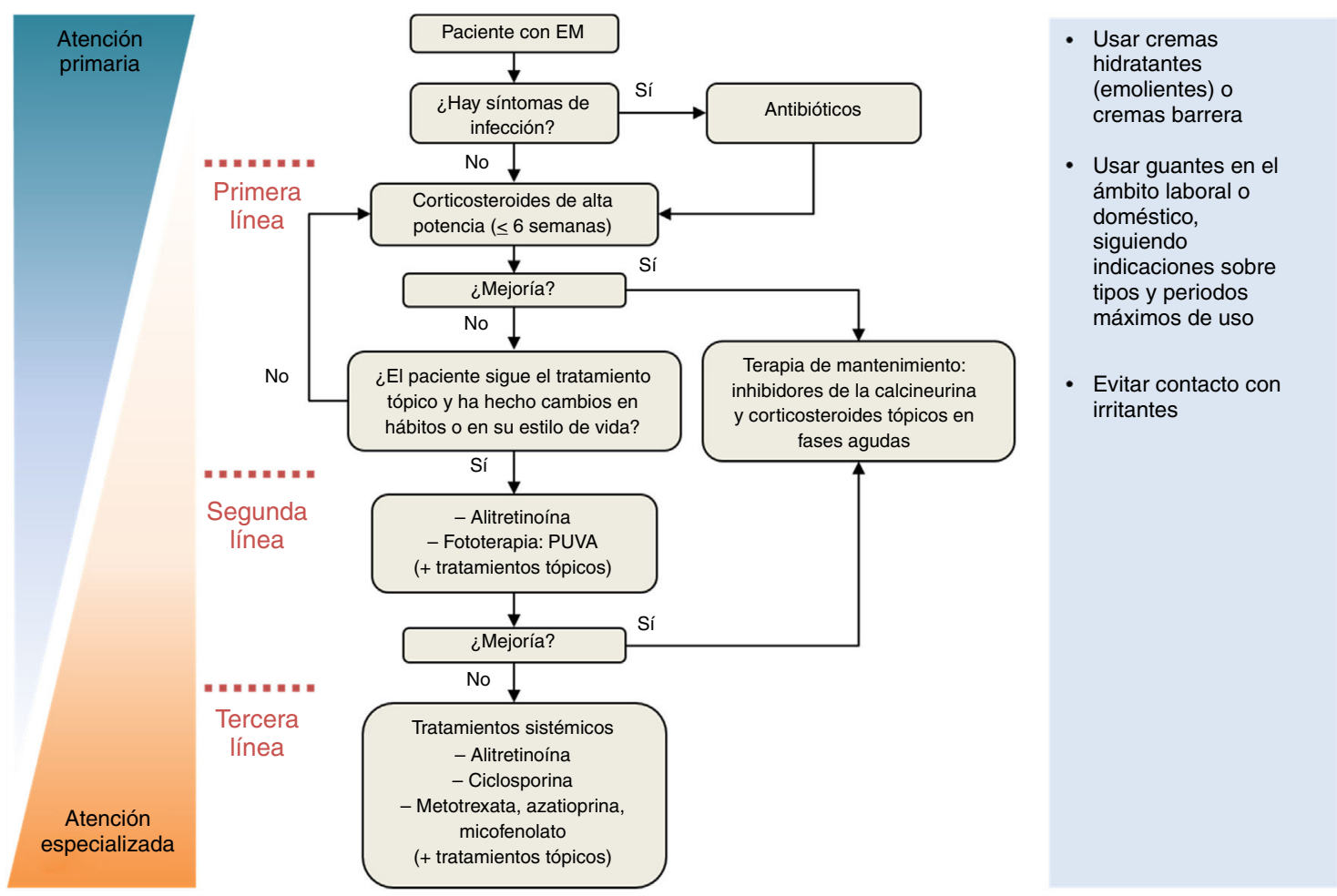

Figura 3 Algoritmo para el tratamiento del eccema de manos (EM). Los cambios en la educación y el estilo de vida, tales como el uso de emolientes y guantes de protección, evitación de irritantes y alérgenos, son obligatorios durante todas las etapas del tratamiento. Se pueden valorar los corticoesteroides orales en caso de exacerbaciones.

Adaptado de De León et al. ${ }^{49}$. 
680 pacientes alemanes adultos con ECM mostró que la alitretinoína fue eficaz en el 56,7\%, independientemente de las formas morfológicas (hiperqueratósicas-fisuradas, de la yema del dedo o vesiculares), y demostrando la eficacia y la tolerabilidad de la alitretinoína en la práctica clínica ${ }^{82}$.

Si bien el tratamiento estándar es de 24 semanas, la alitretinoína sigue siendo bien tolerada para tratamientos de hasta 48 semanas $^{83}$. Un pequeño estudio coreano mostró que la alitretinoína durante solo 12 semanas condujo a una mejoría importante en el $44,4 \%$ de los pacientes con $\mathrm{ECM}^{84}$. La evidencia reciente de la práctica clínica en España también muestra que la alitretinoína es un tratamiento eficaz, a menudo en un solo ciclo $^{85}$

Aunque el tratamiento con alitretinoína es bien tolerado, se han observado efectos adversos dependientes de la dosis, como cefalea ( $20 \%$ de los pacientes con dosis de $30 \mathrm{mg} /$ día), eventos mucocutáneos, hiperlipidemia y alteraciones de la función tiroidea ${ }^{79,80}$. Además de estos eventos adversos, se han notificado en los 6 años posteriores al lanzamiento del producto otros efectos adversos, como náuseas, hipertrigliceridemia, elevación de creatinina-quinasa y depresión ${ }^{86}$. También se deben considerar los efectos adversos a largo plazo de la alitretinoína, ya que este fármaco es teratogénico y su uso debe evitarse 1 mes antes y durante el embarazo en mujeres en edad fértil.

Un estudio reciente mostró que la alitretinoína normaliza la expresión de genes y proteínas de barrera tales como TSLP, claudina 1, loricrina, filagrina y citoqueratina $10^{87}$. Estos cambios se correlacionaron con la eficacia clínica general, lo que sugiere que la alitretinoína exhibe una actividad modificadora de la enfermedad ${ }^{87}$. Se ha formulado la hipótesis de que la alitretinoína podría beneficiar a los pacientes con eccema dishidrótico mediante la regulación de acuaporina 3 y acuaporina $10^{88}$.

Los estudios observacionales de pacientes alemanes con ECM grave mostraron que la alitretinoína producía una mejoría rápida y significativa en la calidad de vida y la productividad laboral, medida por el PGA y diversos índices de calidad de vida ${ }^{89,90}$. Los efectos de la alitretinoína en un grupo de pacientes italianos con ECM mostraron que el fármaco condujo a una mejora notable en la calidad de vida ${ }^{91}$.

\section{Acitretina}

Un estudio retrospectivo mostró que la acitretina es un tratamiento efectivo para el ECM pero que la incidencia de eventos adversos es más alta que con la alitretinoína $(43,1 \% \text { versus } 29,5 \%)^{92}$. Sin embargo, un estudio reciente mostró que una dosis baja de acitretina puede inducir mejoría clínica en pacientes con varios tipos de $\mathrm{EM}^{93}$. Los pacientes con eccema hiperqueratósico de la mano tuvieron con mayor frecuencia un buen efecto del tratamiento con acitretina ${ }^{92}$. Un estudio piloto canadiense demostró que la acitretina era eficaz en el 33,3\% de los pacientes con ECM grave ${ }^{94}$. La acitretina, como la alitretinoína, es un fármaco retinoide $y$, por lo tanto, potencialmente teratogénico. No se recomienda a mujeres con deseo genésico en los siguientes 3 años tras el tratamiento.
Corticoides sistémicos

Las guías de práctica clínica actuales sugieren que el uso de corticosteroides sistémicos debe limitarse al tratamiento del ECM grave durante un máximo de 3 semanas. Sin embargo, no se recomienda para el tratamiento a largo plazo del ECM debido al riesgo de efectos adversos potencialmente peligrosos ${ }^{3}$.

\section{Ciclosporina}

La ciclosporina se ha usado para el tratamiento de la psoriasis, la dermatitis atópica y también para pacientes con ECM que no responden a ningún otro tratamiento ${ }^{3}$. Los primeros estudios en EM demostraron que la eficacia de la ciclosporina oral era similar a la de un CT (beta-metasona17,21-dipropionato) y tenía un efecto equivalente en la mejora de la calidad de vida ${ }^{95,96}$. La eficacia a largo plazo también fue buena, incluso cuando el período de tratamiento es corto ${ }^{97}$. Un estudio retrospectivo reciente mostró que después de 3 meses se registró una buena respuesta al tratamiento en el $62,9 \%$ de los pacientes, especialmente en aquellos con EM vesicular recurrente. La supervivencia de la ciclosporina en pacientes con ECM mostró que tenía una mediana de 10,3 meses. Las razones principales para la interrupción fueron los eventos adversos, especialmente al inicio del tratamiento, y la ineficacia ${ }^{98}$. El tratamiento con ciclosporina debe controlarse cuidadosamente debido a la posibilidad de eventos adversos graves como nefrotoxicidad, riesgo de cáncer, aumento de la presión arterial y mayor riesgo de infección ${ }^{3}$.

\section{Azatioprina}

La evidencia de la eficacia de la azatioprina para el tratamiento del EM es muy limitada, pero podría utilizarse en casos en que otros tratamientos hayan fallado o sean insuficientes ${ }^{3}$. Para optimizar la dosis de azatioprina, mejorar su eficacia clínica y prevenir los efectos secundarios, en algunos pacientes con EM se deben medir inicialmente siempre y más tarde de forma periódica los niveles sanguíneos de nucleótido de 6-tioguanina y 6-metilmercaptopurina metilada, ya que estos metabolitos del fármaco pueden conducir a hepatotoxicidad ${ }^{99}$. La azatioprina se ha probado en la India en el tratamiento de la dermatitis causada por Pharthenium, un alérgeno muy común en este país ${ }^{100}$.

\section{Otros tratamientos}

El metotrexato y el micofenolato se pueden utilizar en tratamientos de segunda y tercera línea del EM sin indicación, pero el nivel de evidencia de estos medicamentos es bajo ${ }^{49,101}$. En un estudio, el tratamiento con metotrexato durante $8-12$ semanas en adultos fue exitoso, pero a menudo seguido de pérdida de efectividad y aparición de efectos adversos, lo que llevó a la interrupción ${ }^{102}$. También su efectividad parece reducida en comparación con otros tratamientos sistémicos, como la acitretina ${ }^{103}$. El metotrexato se demostró eficaz en casos de eccema numular en niños $^{104}$. La oxibutinina es un tratamiento alternativo para la hiperhidrosis y se ha demostrado que mejora el eccema dishidrótico concurrente en algunos $\operatorname{casos}^{105}$. 


\section{Conceptos clave del eccema de manos}

- El EM es una enfermedad multifactorial con una alta prevalencia en la población.

- Es fundamental el establecimiento precoz del diagnóstico y del tratamiento para evitar la posible cronificación.

- El ECM es el EM que se prolonga de forma continua 3 meses o más o recae dos veces o más en un año, a pesar de las medidas terapéuticas adecuadas y la buena adherencia terapéutica.

- No existe una clasificación universalmente aceptada de los diversos tipos de EM, pero se pueden hacer grupos diagnósticos atendiendo a criterios morfológicos y etiológicos.

- El diagnóstico de EM es clínico y se basa en la anamnesis y la exploración física. Las pruebas epicutáneas (parches), prick test, test microbiológicos y biopsias cutáneas son útiles para descartar otras patologías o para establecer un diagnóstico etiológico.

- Ante un caso de EM, el médico de AP debe recomendar la protección adecuada frente a irritantes y humedad, e indicar el uso correcto de guantes y emolientes.

- Cuando el médico de AP identifica un paciente con EM crónico se recomienda valorar la baja laboral y derivar al especialista para la realización de pruebas complementarias.

- Debido a que el EM es una enfermedad que se deriva con frecuencia del ámbito laboral, la prevención de riesgos en ciertas actividades profesionales es esencial.

\section{Financiación}

La elaboración de este documento ha sido financiada por GlaxoSmithKline mediante una beca no condicionada.

\section{Conflicto de intereses}

Los autores declaran no tener ningún conflicto de intereses.

\section{Agradecimientos}

Los autores agradecen el apoyo de Francisco López de Saro en la redacción y la edición de este manuscrito.

\section{Bibliografía}

1. Thyssen JP, Johansen JD, Linneberg A, Menne T. The epidemiology of hand eczema in the general population - prevalence and main findings. Contact Dermatitis. 2010;62:75-87.

2. Alfonso JH, Bauer A, Bensefa-Colas L, Boman A, Bubas M, Constandt $L$, et al. Minimum standards on prevention, diagnosis and treatment of occupational and work-related skin diseases in Europe - Position paper of the cost action standerm (TD 1206). J Eur Acad Dermatol Venereol. 2017;31 Suppl 4:31-43.
3. Diepgen TL, Andersen KE, Chosidow O, Coenraads PJ, Elsner P, English J, et al. Guidelines for diagnosis, prevention and treatment of hand eczema. J Dtsch Dermatol Ges. 2015;13:e1-22.

4. Lynde C, Guenther L, Diepgen TL, Sasseville D, Poulin Y, Gulliver W, et al. Canadian hand dermatitis management guidelines. J Cutan Med Surg. 2010;14:267-84.

5. Menne T, Johansen JD, Sommerlund M, Veien NK. Hand eczema guidelines based on the Danish guidelines for the diagnosis and treatment of hand eczema. Contact Dermatitis. 2011;65:3-12.

6. Coenraads PJ. Hand eczema. N Engl J Med. 2012;36719:1829-37.

7. Diepgen TL, Agner T, Aberer W, Berth-Jones J, Cambazard F, Elsner P, et al. Management of chronic hand eczema. Contact Dermatitis. 2007;57:203-10.

8. Vindenes HK, Svanes C, Lygre SHL, Hollund BE, Langhammer A, Bertelsen RJ. Prevalence of, and work-related risk factors for, hand eczema in a Norwegian general population (the Hunt Study). Contact Dermatitis. 2017;77:214-23.

9. Gronhagen CM, Liden C, Bergstrom A, Kull I, Wahlgren CF, Meding B. Prevalence and incidence of hand eczema in adolescence: Report from BAMSE - a population-based birth cohort. Br J Dermatol. 2014;1713:609-14.

10. Johannisson A, Ponten A, Svensson A. Prevalence, incidence and predictive factors for hand eczema in young adults - a follow-up study. BMC Dermatol. 2013;13:14.

11. Mortz CG, Bindslev-Jensen C, Andersen KE. Hand eczema in the odense adolescence cohort study on atopic diseases and dermatitis (TOACS): Prevalence, incidence and risk factors from adolescence to adulthood. Br J Dermatol. 2014;1712: 313-23.

12. Scalone L, Cortesi PA, Mantovani LG, Belisari A, Ayala F, Fortina $A B$, et al. Clinical epidemiology of hand eczema in patients accessing dermatological reference centres: Results from Italy. Br J Dermatol. 2015;1721:187-95.

13. Apfelbacher C, Molin S, Weisshaar E, Bauer A, Elsner P, Mahler $\mathrm{V}$, et al. Characteristics and provision of care in patients with chronic hand eczema: Updated data from the carpe registry. Acta Derm Venereol. 2014;94:163-7.

14. Minamoto K, Watanabe T, Diepgen TL. Self-reported hand eczema among dental workers in Japan - a cross-sectional study. Contact Dermatitis. 2016;75:230-9.

15. Brans R, Kolomanski K, Mentzel F, Vollmer U, Kaup O, John SM. Colonisation with methicillin-resistant Staphylococcus aureus and associated factors among nurses with occupational skin diseases. Occup Environ Med. 2016;7310:670-5.

16. Heede NG, Thyssen JP, Thuesen BH, Linneberg A, Johansen JD. Predictive factors of self-reported hand eczema in adult danes: A population-based cohort study with 5-year follow-up. Br J Dermatol. 2016;1752:287-95.

17. Gronhagen C, Liden C, Wahlgren CF, Ballardini N, Bergstrom A, Kull I, et al. Hand eczema and atopic dermatitis in adolescents: A prospective cohort study from the BAMSE project. Br J Dermatol. 2015;1735:1175-82.

18. Lee SW, Cheong SH, Byun JY, Choi YW, Choi HY. Occupational hand eczema among nursing staffs in korea: Self-reported hand eczema and contact sensitization of hospital nursing staffs. J Dermatol. 2013;40:182-7.

19. Ibler KS, Jemec GB, Flyvholm MA, Diepgen TL, Jensen A, Agner T. Hand eczema: Prevalence and risk factors of hand eczema in a population of 2274 healthcare workers. Contact Dermatitis. 2012;67:200-7.

20. Ortiz-Salvador JM, Subiabre-Ferrer D, Garcia Rabasco A, Esteve-Martinez A, Zaragoza-Ninet V, Alegre de Miquel V. [Hand eczema in children. Clinical and epidemiological study of the population referred to a tertiary hospital]. An Pediatr (Barc). 2018;88:309-14.

21. Sorensen JA, Fisker MH, Agner T, Clemmensen KK, Ebbehoj NE. Associations between lifestyle factors and hand 
eczema severity: Are tobacco smoking, obesity and stress significantly linked to eczema severity? Contact Dermatitis. 2017;76:138-45.

22. Lai YC, Yew YW. Smoking and hand dermatitis in the united states adult population. Ann Dermatol. 2016;28:164-71.

23. Sorensen JA, Clemmensen KK, Nixon RL, Diepgen TL, Agner T. Tobacco smoking and hand eczema - is there an association? Contact Dermatitis. 2015;73:326-35.

24. Molin S, Ruzicka T, Herzinger T. Smoking is associated with combined allergic and irritant hand eczema, contact allergies and hyperhidrosis. J Eur Acad Dermatol Venereol. 2015;2912:2483-6.

25. Brans R, Skudlik C, Weisshaar E, Gediga K, Scheidt R, Wulfhorst $B$, et al. Association between tobacco smoking and prognosis of occupational hand eczema: A prospective cohort study. $\mathrm{Br}$ J Dermatol. 2014;1715:1108-15.

26. Lukacs J, Schliemann S, Elsner P. Association between smoking and hand dermatitis - a systematic review and meta-analysis. J Eur Acad Dermatol Venereol. 2015;29:1280-4.

27. Meding B, Anveden Berglind I, Alderling M, Lindahl G, Wrangsjo $\mathrm{K}$. Water exposure - challenging differences between occupations. Contact Dermatitis. 2016;74:22-8.

28. Visser MJ, Verberk MM, van Dijk FJ, Bakker JG, Bos JD, Kezic S. Wet work and hand eczema in apprentice nurses; Part I of a prospective cohort study. Contact Dermatitis. 2014;70:44-55.

29. Fartasch M. Wet work and barrier function. Curr Probl Dermatol. 2016;49:144-51.

30. Nichol K, Copes R, Spielmann S, Kersey K, Eriksson J, Holness DL. Workplace screening for hand dermatitis: A pilot study. Occup Med (Lond). 2016;66:46-9.

31. Van der Meer EW, Boot CR, van der Gulden JW, Jungbauer FH, Coenraads PJ, Anema JR. Hand eczema among healthcare professionals in the netherlands: Prevalence, absenteeism, and presenteeism. Contact Dermatitis. 2013;69:164-71.

32. Ibler KS, Jemec GB, Garvey LH, Agner T. Prevalence of delayed-type and immediate-type hypersensitivity in healthcare workers with hand eczema. Contact Dermatitis. 2016;75:223-9.

33. Foss-Skiftesvik MH, Winther L, Johnsen CR, Zachariae C, Johansen JD. Incidence of skin and respiratory diseases among danish hairdressing apprentices. Contact Dermatitis. 2017;76: 160-6.

34. Schwensen JF, Johansen JD, Veien NK, Funding AT, Avnstorp C, Osterballe M, et al. Occupational contact dermatitis in hairdressers: An analysis of patch test data from the danish contact dermatitis group, 2002-2011. Contact Dermatitis. 2014;70:233-7.

35. Steengaard SS, Bregnhoj A, Johansen JD. Hand eczema among hairdressing apprentices in denmark following a nationwide prospective intervention programme: 6-year follow-up. Contact Dermatitis. 2016;75:32-40.

36. Aktas E, Esin MN. Skin disease symptoms and related risk factors among young workers in high-risk jobs. Contact Dermatitis. 2016;75:96-105.

37. Samardzic T, Varnai VM, Bakotic M, Babic Z, Brans R, Cvijetic Avdagic S, et al. Skin health and safety at work in croatian hairdressing apprentices. Contact Dermatitis. 2016;75: 25-31.

38. Bhatia R, Sharma VK, Ramam M, Sethuraman G, Yadav CP. Clinical profile and quality of life of patients with occupational contact dermatitis from New Delhi, India. Contact Dermatitis. 2015;73:172-81.

39. Crane MM, Webb DJ, Watson E, Cunliffe T, English J. Hand eczema and steroid-refractory chronic hand eczema in general practice: Prevalence and initial treatment. $\mathrm{Br} \mathrm{J}$ Dermatol. 2017;1764:955-64.

40. Mollerup A, Veien NK, Johansen JD. An analysis of gender differences in patients with hand eczema - everyday exposures, severity, and consequences. Contact Dermatitis. 2014;71:21-30.

41. Kasemsarn P, Bosco J, Nixon RL. The role of the skin barrier in occupational skin diseases. Curr Probl Dermatol. 2016;49:135-43.

42. Molin S, Merl J, Dietrich KA, Regauer M, Flaig M, Letule V, et al. The hand eczema proteome: Imbalance of epidermal barrier proteins. Br J Dermatol. 2015;1724:994-1001.

43. Kohli N, Nedorost S. Inflamed skin predisposes to sensitization to less potent allergens. J Am Acad Dermatol. 2016;75, 312.e1317.e1.

44. Mernelius S, Carlsson E, Henricson J, Lofgren S, Lindgren PE, Ehricht R, et al. Staphylococcus aureus colonization related to severity of hand eczema. Eur J Clin Microbiol Infect Dis. 2016;35:1355-61.

45. Schiattarella M, Patruno C, Balato N, Canta L, Caiazzo G, Lembo S, et al. Chronic hand eczema: Is il-36alpha helpful in diagnosis and classification? G Ital Dermatol Venereol. 2017; 1526:578-85.

46. Johansen JD, Hald M, Andersen BL, Laurberg G, Danielsen A, Avnstorp C, et al., Classification of hand eczema: Clinical and aetiological types. Based on the guideline of the danish contact dermatitis group. Contact Dermatitis. 2011;65:13-21.

47. Boonstra MB, Christoffers WA, Coenraads PJ, Schuttelaar ML. Patch test results of hand eczema patients: Relation to clinical types. J Eur Acad Dermatol Venereol. 2015;29:940-7.

48. Diepgen TL, Andersen KE, Brandao FM, Bruze M, Bruynzeel DP, Frosch P, et al. Hand eczema classification: A cross-sectional, multicentre study of the aetiology and morphology of hand eczema. Br J Dermatol. 2009;1602:353-8.

49. De León FJ, Berbegal L, Silvestre JF. Management of chronic hand eczema. Actas Dermosifiliogr. 2015;1067:533-44.

50. Agner T, Aalto-Korte K, Andersen KE, Foti C, Gimenez-Arnau A, Goncalo M, et al. Classification of hand eczema. J Eur Acad Dermatol Venereol. 2015;2912:2417-22.

51. Schwensen JF, Menne T, Johansen JD. The combined diagnosis of allergic and irritant contact dermatitis in a retrospective cohort of 1000 consecutive patients with occupational contact dermatitis. Contact Dermatitis. 2014;71:356-63.

52. Aquino $M$, Fonacier $L$. The role of contact dermatitis in patients with atopic dermatitis. J Allergy Clin Immunol Pract. 2014;2:382-7.

53. Owen JL, Vakharia PP, Silverberg JI. The role and diagnosis of allergic contact dermatitis in patients with atopic dermatitis. Am J Clin Dermatol. 2018;19:293-302.

54. Weisshaar E, Kallen U, Weiss M. "The itching hand" - important differential diagnoses and treatment. J Dtsch Dermatol Ges. 2013;11:31-42.

55. Chen JK, Jacob SE, Nedorost ST, Hanifin JM, Simpson EL, Boguniewicz $M$, et al. A pragmatic approach to patch testing atopic dermatitis patients: Clinical recommendations based on expert consensus opinion. Dermatitis. 2016;27:186-92.

56. Kostner L, Anzengruber F, Guillod C, Recher M, SchmidGrendelmeier P, Navarini AA. Allergic contact dermatitis. Immunol Allergy Clin North Am. 2017;37:141-52.

57. Geier J, Krautheim A, Uter W, Lessmann H, Schnuch A. Occupational contact allergy in the building trade in germany: Influence of preventive measures and changing exposure. Int Arch Occup Environ Health. 2011;84:403-11.

58. Uter W, Geier J, Lessmann H, Schnuch A. Is contact allergy to glyceryl monothioglycolate still a problem in Germany? Contact Dermatitis. 2006;55:54-6.

59. Crepy MN, Lecuen J, Ratour-Bigot C, Stocks J, Bensefa-Colas L. Accelerator-free gloves as alternatives in cases of glove allergy in healthcare workers. Contact Dermatitis. 2018;78:28-32.

60. Van der Meer EW, Boot CR, Twisk JW, Coenraads PJ, Jungbauer $\mathrm{FH}$, van der Gulden JW, et al. Hands4U: The effectiveness of a multifaceted implementation strategy on behaviour 
related to the prevention of hand eczema-a randomised controlled trial among healthcare workers. Occup Environ Med. 2014;71:492-9.

61. Van der Meer EW, Boot CR, van der Gulden JW, Knol DL, Jungbauer FH, Coenraads PJ, et al. Hands4U: The effects of a multifaceted implementation strategy on hand eczema prevalence in a healthcare setting. Results of a randomized controlled trial. Contact Dermatitis. 2015;72:312-24.

62. Van der Meer EW, van Dongen JM, Boot CR, van der Gulden JW, Bosmans JE, Anema JR, Economic evaluation of a multifaceted implementation strategy for the prevention of hand eczema among healthcare workers in comparison with a control group: The Hands4U study. Acta Derm Venereol. 2016;96:499-504.

63. English J, Aldridge R, Gawkrodger DJ, Kownacki S, Statham B, White JM, et al. Consensus statement on the management of chronic hand eczema. Clin Exp Dermatol. 2009;34:761-9.

64. Wei J, Gerlich J, Vogelberg C, von Mutius E, Windstetter D, Genuneit J, et al. Do young adults with atopic dermatitis avoid harmful workplace exposure at their first job? A prospective cohort study. Int Arch Occup Environ Health. 2016;89:397-406.

65. Wilke A, Gediga G, Schlesinger T, John SM, Wulfhorst B. Sustainability of interdisciplinary secondary prevention in patients with occupational hand eczema: A 5-year follow-up survey. Contact Dermatitis. 2012;67:208-16.

66. Loden M, Wiren K, Smerud K, Meland N, Honnas H, Mork $\mathrm{G}$, et al. Treatment with a barrier-strengthening moisturizer prevents relapse of hand-eczema. An open, randomized, prospective, parallel group study. Acta Derm Venereol. 2010;90:602-6.

67. Eichenfield LF, Tom WL, Berger TG, Krol A, Paller AS, Schwarzenberger K, et al. Guidelines of care for the management of atopic dermatitis: Section 2. Management and treatment of atopic dermatitis with topical therapies. J Am Acad Dermatol. 2014;71:116-32.

68. Brans R, Skudlik C, Weisshaar E, Scheidt R, Ofenloch R, Elsner P, et al. Multicentre cohort study "Rehabilitation of occupational skin diseases - optimization and quality assurance of inpatient management (ROQ)"': Results from a 3-year follow-up. Contact Dermatitis. 2016;75:205-12.

69. Weisshaar E, Skudlik C, Scheidt R, Matterne U, Wulfhorst B, Schonfeld M, et al. Multicentre study "Rehabilitation of occupational skin diseases - optimization and quality assurance of inpatient management (ROQ)" - Results from 12-month follow-up. Contact Dermatitis. 2013;68:169-74.

70. Berbegal L, DeLeon FJ, Silvestre JF. Hipersensitivity reactions to corticosteroids. Actas Dermosifiliogr. 2016;1072:107-15.

71. Loden M, Wiren K, Smerud KT, Meland N, Honnas H, Mork G, et al. The effect of a corticosteroid cream and a barrierstrengthening moisturizer in hand eczema. A double-blind, randomized, prospective, parallel group clinical trial. J Eur Acad Dermatol Venereol. 2012;26:597-601.

72. Jungersted JM, Hogh JK, Hellegren LI, Jemec GB, Agner T. Effects of topical corticosteroid and tacrolimus on ceramides and irritancy to sodium lauryl sulphate in healthy skin. Acta Derm Venereol. 2011;91:290-4.

73. Shroff A, Malajian D, Czarnowicki T, Rose S, Bernstein DM, Singer GK, et al. Use of $308 \mathrm{~nm}$ excimer laser for the treatment of chronic hand and foot eczema. Int J Dermatol. 2016;55:e447-53.

74. Blair HA, Scott LJ. Alitretinoin: A review in severe chronic hand eczema. Drugs. 2016;7613:1271-9.

75. King T, McKenna J, Alexandroff AB. Alitretinoin for the treatment of severe chronic hand eczema. Patient Prefer Adherence. 2014;8:1629-34.

76. Schmith GD, Singh R, Gomeni R, Graff O, Hamedani AG, Troughton JS, et al. Use of longitudinal dose-response modeling to support the efficacy and tolerability of alitretinoin in severe refractory chronic hand eczema (CHE). CPT Pharmacometrics Syst Pharmacol. 2015;4:255-62.

77. Ham K, Maini P, Gooderham MJ. Real-world experience with alitretinoin in a community dermatology practice setting in patients with chronic hand dermatitis. J Cutan Med Surg. 2014;18:332-6.

78. Gola M, Milanesi N, d'Erme AM. Clinical evaluation and assessment of the therapeutic efficacy of alitretinoin in a group of patients with chronic hand eczema refractory to topical steroid therapy. G Ital Dermatol Venereol. 2014;1494: 435-9.

79. Ruzicka T, Lynde CW, Jemec GB, Diepgen T, Berth-Jones J, Coenraads PJ, et al. Efficacy and safety of oral alitretinoin (9cis retinoic acid) in patients with severe chronic hand eczema refractory to topical corticosteroids: Results of a randomized, double-blind, placebo-controlled, multicentre trial. Br J Dermatol. 2008;1584:808-17.

80. Fowler JF, Graff O, Hamedani AG. A phase 3, randomized, double-blind, placebo-controlled study evaluating the efficacy and safety of alitretinoin (BAL4079) in the treatment of severe chronic hand eczema refractory to potent topical corticosteroid therapy. J Drugs Dermatol. 2014;1310:1198-204.

81. Bissonnette R, Worm M, Gerlach B, Guenther L, Cambazard $F$, Ruzicka $T$, et al. Successful retreatment with alitretinoin in patients with relapsed chronic hand eczema. Br J Dermatol. 2010;1622:420-6.

82. Diepgen TL, Pfarr E, Zimmermann T. Efficacy and tolerability of alitretinoin for chronic hand eczema under daily practice conditions: Results of the toccata open study comprising 680 patients. Acta Derm Venereol. 2012;92:251-5.

83. Lynde C, Cambazard F, Ruzicka T, Sebastian M, Brown TC, Maares J. Extended treatment with oral alitretinoin for patients with chronic hand eczema not fully responding to initial treatment. Clin Exp Dermatol. 2012;37:712-7.

84. Kwon HI, Kim JE, Ko JY, Ro YS. Efficacy and safety of alitretinoin for chronic hand eczema in korean patients. Ann Dermatol. 2016;28:364-70.

85. Urrutia S, Roustan G, Plazas MJ, Armengol S, Paz S, Lizan $\mathrm{L}$. Oral alitretinoin in the treatment of severe refractory chronic hand eczema in the spanish national health system: Description and analysis of current clinical practice. Actas Dermosifiliogr. 2016;1072:142-8.

86. Morris M, Schifano L, Fong R, Graff O. Safety of alitretinoin for severe refractory chronic hand eczema: Clinical studies and postmarketing surveillance. J Dermatolog Treat. 2016;27:54-8.

87. Kumari V, Timm K, Kuhl AA, Heine G, Worm M. Impact of systemic alitretinoin treatment on skin barrier gene and protein expression in patients with chronic hand eczema. Br J Dermatol. 2016;1756:1243-50.

88. Soler DC, Bai X, Ortega L, Pethukova T, Nedorost ST, Popkin DL, et al. The key role of aquaporin 3 and aquaporin 10 in the pathogenesis of pompholyx. Med Hypotheses. 2015;84:498-503.

89. Augustin M, Thaci D, Kamps A. Impact on quality of life of alitretinoin in severe chronic hand eczema: FUGETTA real-world study. J Dtsch Dermatol Ges. 2016;1412:1261-70.

90. Thaci D, Augustin M, Westermayer B, Kamps A, Hennig M. Effectiveness of alitretinoin in severe chronic hand eczema: Passion, a real-world observational study. J Dermatolog Treat. 2016;27:577-83.

91. Gola M, d'Erme AM, Milanesi N, Marmugi L. Effects of alitretinoin on quality of life of patients having chronic hand eczema: An observational study. Dermatitis. 2013;24:166-9.

92. Politiek K, Christoffers WA, Coenraads PJ, Schuttelaar MA. Alitretinoin and acitretin in severe chronic hand eczema; results from a retrospective daily practice study. Dermatol Ther. 2016;29:364-71. 
93. Song M, Lee HJ, Lee WK, Kim HS, Ko HC, Kim MB, et al. Acitretin as a therapeutic option for chronic hand eczema. Ann Dermatol. 2017;29:385-7.

94. Tan J, Maari C, Nigen S, Bolduc C, Bissonnette R. Open-label exploratory study of acitretin for the treatment of severe chronic hand dermatitis. J Dermatolog Treat. 2015;26:373-5.

95. Granlund H, Erkko P, Eriksson E, Reitamo S. Comparison of cyclosporine and topical betamethasone-17,21-dipropionate in the treatment of severe chronic hand eczema. Acta Derm Venereol. 1996;76:371-6.

96. Granlund H, Erkko P, Reitamo S. Comparison of the influence of cyclosporine and topical betamethasone-17,21-dipropionate treatment on quality of life in chronic hand eczema. Acta Derm Venereol. 1997;77:54-8.

97. Granlund H, Erkko P, Reitamo S. Long-term follow-up of eczema patients treated with cyclosporine. Acta Derm Venereol. 1998;78:40-3.

98. Christoffers WA, Politiek K, Coenraads PJ, van der Schaft J, de Bruin-Weller MS, Schuttelaar ML. Drug survival of cyclosporine in the treatment of hand eczema: A multicentre, daily use study. J Eur Acad Dermatol Venereol. 2016;30:63-6.

99. Garritsen FM, van der Schaft J, Bruijnzeel-Koomen CAF, van Schaik RH, de Graaf M, van den Broek MPH, et al. Thiopurine metabolite levels in patients with atopic dermatitis and/or chronic hand/foot eczema treated with azathioprine. J Dermatolog Treat. 2018;29:375-82.

100. Verma KK, Sethuraman G, Kalavani M. Weekly azathioprine pulse versus daily azathioprine in the treatment of parthenium dermatitis: A non-inferiority randomized controlled study. Indian J Dermatol Venereol Leprol. 2015;81: 251-6.

101. Halling-Overgaard AS, Zachariae C, Thyssen JP. Management of atopic hand dermatitis. Dermatol Clin. 2017;35:365-72.

102. Politiek K, van der Schaft J, Christoffers WA, Coenraads PJ, van den Reek JM, de Jong EM, et al. Drug survival of methotrexate treatment in hand eczema patients: Results from a retrospective daily practice study. J Eur Acad Dermatol Venereol. 2016;30:1405-7.

103. O'Shea PM, Lugo-Somolinos A. Methotrexate versus acitretin in the treatment of chronic hand dermatitis. J Drugs Dermatol. 2015;1412:1389-91.

104. Roberts H, Orchard D. Methotrexate is a safe and effective treatment for paediatric discoid (nummular) eczema: A case series of 25 children. Australas J Dermatol. 2010;51:128-30.

105. Markantoni V, Kouris A, Armyra K, Vavouli C, Kontochristopoulos G. Remarkable improvement of relapsing dyshidrotic eczema after treatment of coexistant hyperhidrosis with oxybutynin. Dermatol Ther. 2014;27:365-8. 